\title{
Hertel Exophthalmometry and Computed Tomography for the Evaluation of Exophthalmos in Patients with Thyroid-Associated Ophthalmopathy
}

\author{
Nina Staneva Stoyanova, Marieta Konareva-Kostianeva, Vesela Mitkova-Hristova \\ Department of Ophthalmology, Faculty of Medicine, Medical University of Plovdiv, Plovdiv, Bulgaria \\ Email: nina.st.st@abv.bg
}

How to cite this paper: Stoyanova, N.S., Konareva-Kostianeva, M. and Mitkova-Hristova, V. (2020) Hertel Exophthalmometry and Computed Tomography for the Evaluation of Exophthalmos in Patients with Thyroid-Associated Ophthalmopathy. Open Journal of Ophthalmology, 10, 164-173. https://doi.org/10.4236/ojoph.2020.102018

Received: April 27, 2020

Accepted: May 15, 2020

Published: May 18, 2020

Copyright $\odot 2020$ by author(s) and Scientific Research Publishing Inc. This work is licensed under the Creative Commons Attribution International License (CC BY 4.0).

http://creativecommons.org/licenses/by/4.0/

\begin{abstract}
Objective: To compare the values of exophthalmos measured by computed tomography (CT) and Hertel exophthalmometry (HE) in patients with thyroid-associated ophthalmopathy (TAO). Material and Methods: One hundred and seventy eyes were examined in 85 patients with TAO. Each patient underwent a complete ophthalmic examination, Hertel exophthalmometry, and CT of the orbits through a 16-slice CT scanner (Bright Speed, General Electric), measuring the extraocular muscles, the total muscle thickness sum (MTS), and proptosis. The patients were divided into two groups-with activity and without TAO activity, the activity being assessed by means of the Clinical Activity Score (CAS) and the severity-according to the EUGOGO classification. Results: TAO activity was detected in 45 patients (90 eyes, $53 \%$ ) with MTS of $23.54 \pm 5.73 \mathrm{~mm}$, IOP of $19.78 \pm 4.49 \mathrm{~mm} \mathrm{Hg}$, Hertel exophthalmos of $23.08 \pm 4.19 \mathrm{~mm}$ and measured by CT $-23.32 \pm 4.33 \mathrm{~mm}$. Forty patients ( 80 eyes, $47 \%$ ) were without TAO activity, with MTS of $19.28 \pm$ 4.03 , IOP of $16.6 \pm 4.51 \mathrm{~mm} \mathrm{Hg}$, Hertel exophthalmos of $20.03 \pm 3.84 \mathrm{~mm}$ and measured by CT $-19.84 \pm 4.47 \mathrm{~mm}$. A correlation was detected between exophthalmos and: MTS, IOP, the activity and severity of TAO. High congruence was established between the two methods of measuring exophthalmos-CT and HE (Pearson correlation, $r=0.690, p=0.000$ ). Conclusion: Our results showed a high degree of consistency between Hertel exophthalmometry and multidetector CT for the evaluation of exophthalmos in patients with TAO. Exophthalmos is an important clinical feature and its measurement and monitoring over time assess the clinical course and outcome of treatment.
\end{abstract}




\section{Keywords}

Thyroid-Associated Ophthalmopathy, Hertel Exophthalmometry, Computed Tomography

\section{Introduction}

Thyroid-associated ophthalmopathy is the most common extrathyroidal manifestation of Graves' disease and the hyperthyroidism related to it. In rare cases, it is observed in patients with Hashimoto's thyroiditis, hypothyroidism or euthyroidism [1]. TAO is a chronic autoimmune process against orbital antigens, in which lymphocytic infiltration, fibroblast proliferation and adipogenesis develop, along with the accumulation of glucosaminoglycans in the tissues of the orbit and an increase in their volume, with typical fusiform thickening of the extraocular muscles and the eyeball being pushed forward as a sign of spontaneous decompression. The pathological process goes through two consecutive phases-an active inflammatory phase, which lasts from 6 months to 5 years, and an inactive fibrotic phase. The use of the Clinical Activity Score (CAS) allows for an assessment of the disease activity [2]. The clinical manifestations of TAO include eyelid retraction, exophthalmos, edema and hyperemia of the eyelids and conjunctiva, pain in the orbits, as well as eye movement pain and low vision.

A classic method of clinical evaluation of eye protrusion is Hertel exophthalmometry. Different types of exophthalmometers exist and there is some subjectivity in reading the values. In some patients with severe eyelid edema, ptosis, strabismus, and poor cooperation, it is difficult to measure the Hertel exophthalmos. Another method of determining proptosis, in which the possible measurement errors are considerably fewer and which provides an objective estimate, is the computed tomography of the orbits. CT scans are widely available and demonstrate high repeatability and reproducibility.

The purpose of the present study was to compare multidetector computed tomography and Hertel exophthalmometry in order to evaluate proptosis in patients with thyroid-associated ophthalmopathy.

\section{Material and Methods}

This prospective study included 85 patients with TAO (170 orbits), examined at the University Eye Clinic in the period 2012-2019. The diagnosis of TAO was established on the basis of clinical features, CT-changes and clinical laboratory tests in patients with a history of thyroid disease. As exclusion criteria were viewed patients with thyroid disease without $\mathrm{TAO}$, the presence of another inflammatory or space-occupying process or traumas leading to exophthalmos; excessive myopia or orbital decompression performed prior to the study. A detailed medical history was taken in all patients and a complete ophthalmologic examination and consultation with an endocrinologist were performed. All patients underwent Hertel exophthalmometry, CT of orbits, a Goldmann eye pres- 
sure examination and an examination of the peripheral vision of both eyes (Humphrey Field Analyzer 30-2, Full Threshold). To evaluate thyroid function at the time of the study, TSH, T3, T4, anti-TG, anti-TRO, TRAb levels were examined. The disease activity was determined using the Clinical Activity Score (Mourits, 1989), based on the presence of the classic symptoms of inflammation: pain ( 2 points), hyperemia ( 2 points), edema ( 4 points), dysfunction ( 2 points). A score of 3 or more points defined the status as active, and a score of 2 or less than 2-as inactive [2].

The EUGOGO classification was used to evaluate the severity of TAO, dividing patients into 3 categories: 1 ) severe, sight-threatening; 2 ) moderate to severe; 3) mild degree [3] (Table 1).

The same mirror Hertel exophthalmometer (Oculus) was used in all patients, and the study was performed by one examiner. The test complied with the following rules: the exophthalmometer was positioned in such a way that the concave sections of the two frames touched the outer rims of the orbits at the lowest point. During the examination, the patient looked straight ahead and fixed the opposing eye of the examiner, and the visual axis of his/her other eye was blocked by the physician's thumb, the examinee and examiner's eyes being aligned in one plane. The examiner detected the exophthalmos with one eye only: the right eye-to evaluate the exophthalmos of the patient's left eye and vice versa. Which division of the scale the corneal apex corresponded to for each eye was reported, as well as the distance at which the test was performed along the divisions of the rail (base).

Multidetector orbital computed tomography (MDCT) was performed using a 16-slice CT (Bright Speed, GE) according to standard orbital examination protocols for adult patients. All CT examinations were carried out without the use of intravenous contrast. In the axial slices, the thickness of the horizontal muscles (medial rectus muscle (MRM) and lateral rectus muscle (LRM) was measured in millimetres, and in the coronary sections-the thickness of the inferior rectus muscle (IRM) and superior muscular complex (SMC) including superior rectus muscle and superior oblique muscle were measured. All muscles were examined on different scans, taking into account their largest size. The muscle thickness sum (total muscle thickness) of each eye was measured in millimetres (MTS = MRM + LRM + SMC + IRM). The proptosis of each eye was examined in axial sections along the perpendicular distance between the anterior pole of the eye and the interzygomatic line (Figure 1).

The statistical analysis was carried out using the SPSS program, version 23. A descriptive analysis was employed to describe the indices, presented by an arithmetic average and standard deviation. The Kolmogorov-Smirnov test was used to determine the type of quantitative trait distribution, the Independent Samples T-test to compare the values between two groups, and the One-Way ANOVA to compare the values between multiple groups. A value of $\mathrm{p}<0.05$ was considered significant (with a 95\% confidence interval). Pearson's correlation coefficient was used to estimate a linear association. The Bland-Altman graph was used to evaluate the persistent and systematic deviation between the exophthalmos measuring methods. 
Table 1. TAO severity.

\begin{tabular}{|c|c|}
\hline Severity & Signs \\
\hline 1) Sight-threatening & Patients with dysthyroid optic neuropathy (DON) and or corneal breakdown. \\
\hline GO (severe) & This category warrants immediate intervention. \\
\hline $\begin{array}{l}\text { 2) Moderate to } \\
\text { severe GO }\end{array}$ & $\begin{array}{l}\text { Patients without sight-threatening GO whose eye disease has sufficient } \\
\text { impact on daily life to justify the risks of immunosuppression (if active) or } \\
\text { surgical intervention (if inactive). Patients with moderate to severe GO } \\
\text { usually have any one or more of the following: lid retraction }>2 \mathrm{~mm} \text {, } \\
\text { moderate or severe soft tissue involvement, exophthalmos }>3 \mathrm{~mm} \text { above } \\
\text { normal for race and gender, inconstant or constant diplopia. }\end{array}$ \\
\hline & $\begin{array}{l}\text { Patients whose features of GO have only a minor impact on daily life } \\
\text { insufficient to justify immunosuppressive or surgical treatment. They usually }\end{array}$ \\
\hline 3) Mild GO & $\begin{array}{l}\text { only have one or more of the following: minor lid retraction }(<2 \mathrm{~mm}) \text {, mild } \\
\text { soft tissue involvement, exophthalmos }<3 \mathrm{~mm} \text { above normal for race and } \\
\text { gender, transient or no diplopia, corneal exposure responsive to lubricants. }\end{array}$ \\
\hline
\end{tabular}

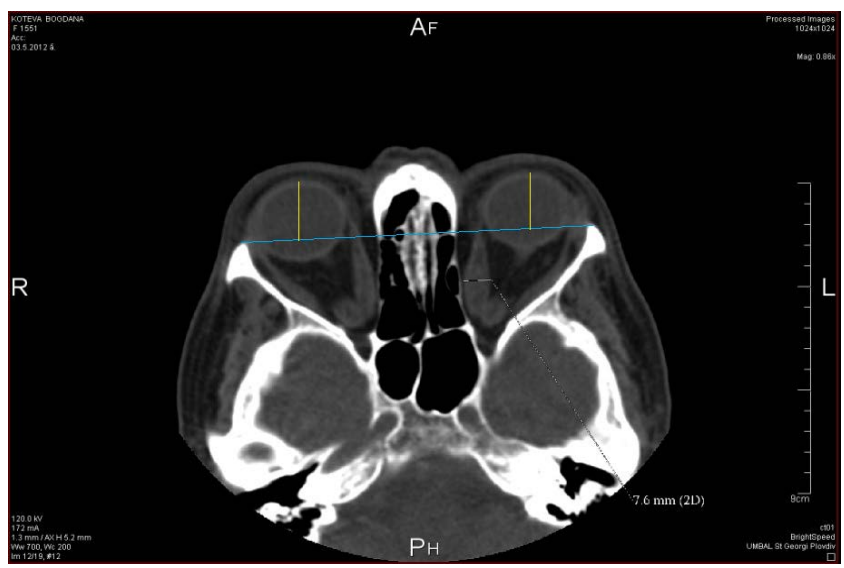

Figure 1. CT measurement of exophthalmos according to the distance (perpendicular) of the anterior pole of the eye (yellow line) to the interzygomatic line (marked in blue).

All patients included in the study were Caucasian and had signed an informed consent to participate in the study.

\section{Results}

The average age of the study group of patients with TAO was $49.36 \pm 11.0$ years old (between 22 and 72). Nineteen patients were male (22.4\%) (average age: $49.84 \pm 9.58)$ and 66 were female $(77.6 \%)$ (average age: $49.23 \pm 11.53$ ). Thirty-two patients (37.6\%) had hyperthyroidism, 10 (11.8\%) had hypothyroidism and $43(50.6 \%)$ had euthyroidism. The majority of patients suffered from Graves' disease-71 (83.5\%), and 13 had autoimmune thyroiditis (15.3\%), only 1 patient having primary hypothyroidism (1.2\%). The mean duration of TAO was $62.89 \pm$ 77 months.

The measurements of Hertel exophthalmos and CT across the study group of patients, for both sexes separately and according to their age, are given in Table 2. 
Both methods of assessment of exophthalmos established statistically higher values of exophthalmos in men than in women (Independent Samples T-test, $\mathrm{p}<$ 0.001). No statistically significant difference in the values of exophthalmos, measured by both methods, was found between the patients in the different age groups (Independent Samples T-test, $\mathrm{p}>0.05$ ).

Disease activity was detected in 45 patients (53\%), while 40 (47\%) showed none. The distribution of patients according to severity classification is presented in Table 3.

Table 3 shows the values of exophthalmos measured by HE and CT in patients according to the activity and severity of the disease.

We found statistically significantly higher values of exophthalmos, measured by both methods, in patients with disease activity rather than in patients without activity (Independent Samples T-test, $\mathrm{p}<0.001$ ), as well as higher values in patients with severe and moderate severity of TAO (ANOVA, LSD, p < 0.05).

Descriptive statistics on the thickness of extraocular muscles and the muscle thickness sum (total muscle thickness) are presented in Table 4. We established a significant positive mean value correlation between the exophthalmos values and MTS in TAO patients by both methods (for HE $r=0.480, p<0.001$; for CT $\mathrm{r}=0.571, \mathrm{p}<0.001)$. With the increase of MTS values, exophthalmos is also on the rise (Figure 2). In patients with disease activity, we measured higher values of MTS $(23.54 \pm 5.73 \mathrm{~mm})$ compared to those without activity $(19.29 \pm 4.03$ $\mathrm{mm}$ ) (Independent Samples T-test, $\mathrm{p}<0.001$ ).

We established a positive correlation, although of low magnitude, between the values of exophthalmos and IOP_for HE $\mathrm{r}=0.279, \mathrm{p}<0.001$; for $\mathrm{CT} \mathrm{r}=0.354$, $\mathrm{p}<0.001$. In patients with greater exophthalmos, measured by both methods, higher IOP values were established (Figure 3 ). The average value of IOP was $18.28 \pm 4.76 \mathrm{~mm} \mathrm{Hg}$, while patients with disease activity showed higher values $(19.78 \pm 4.49 \mathrm{mmHg})$ compared to those without TAO activity $(16.6 \pm 4.51$ $\mathrm{mmHg})$.

We established a high degree of consistency between the two methods of examining exophthalmos in patients with TAO (Pearson correlation, $r=0.690, p=$ 0.000 ; for the right eye $r=0.730, p=0.000$; for the left eye $r=0.655, p=0.000$ ). Figure 4 shows the Bland-Altman graph demonstrating the consistency of the two methods.

Table 2. Hertel and CT exophthalmometry in patients with TAO by gender and age.

\begin{tabular}{ccc}
\hline Patients & Hertel, mm & CT, mm \\
\hline Total & $21.66 \pm 4.30$ & $21.68 \pm 4.7$ \\
Men & $24.03 \pm 4.22$ & $23.43 \pm 4.83$ \\
Women & $20.96 \pm 4.08$ & $21.18 \pm 4.58$ \\
Age up to 40 years old & $21.93 \pm 4.11$ & $21.54 \pm 3.56$ \\
Age above 40 years old & $21.57 \pm 4.37$ & $21.73 \pm 5.03$ \\
\hline
\end{tabular}


Table 3. Hertel and CT-exophthalmometry according to the activity and severity of TAO.

\begin{tabular}{cccc}
\hline Index & Number of Eyes, $\mathrm{n}(\%)$ & Hertel, mm & CT, mm \\
\hline With activity & $90(53 \%)$ & $23.08 \pm 4.19$ & $23.32 \pm 4.33$ \\
Without activity & $80(47 \%)$ & $20.03 \pm 3.84$ & $19.84 \pm 4.47$ \\
Severe Degree & $12(7.05 \%)$ & $26.0 \pm 5.15$ & $26.31 \pm 6.94$ \\
Moderate Degree & $54(31.76 \%)$ & $24.09 \pm 3.33$ & $23.24 \pm 3.51$ \\
Mild Degree & $104(61.18 \%)$ & $19.89 \pm 3.65$ & $20.34 \pm 4.40$ \\
\hline
\end{tabular}

Table 4. Thickness of the extraocular muscles and muscle thickness sum in patients with TAO.

\begin{tabular}{cccc}
\hline Muscle & Number of eyes & Mean \pm SD, mm & Minimum - maximum value, mm \\
\hline Medial rectus & 170 & $5.73 \pm 2.03$ & $2.8-13$ \\
Lateral rectus & 170 & $4.49 \pm 1.6$ & $1.8-9.9$ \\
Superior muscular complex & 170 & $5.48 \pm 1.6$ & $1.2-11.4$ \\
Inferior rectus & 170 & $5.95 \pm 1.84$ & $2.1-12.0$ \\
Muscle thickness sum & 170 & $21.62 \pm 5.61$ & $12.4-42.9$ \\
(MTS) & & & \\
\hline
\end{tabular}

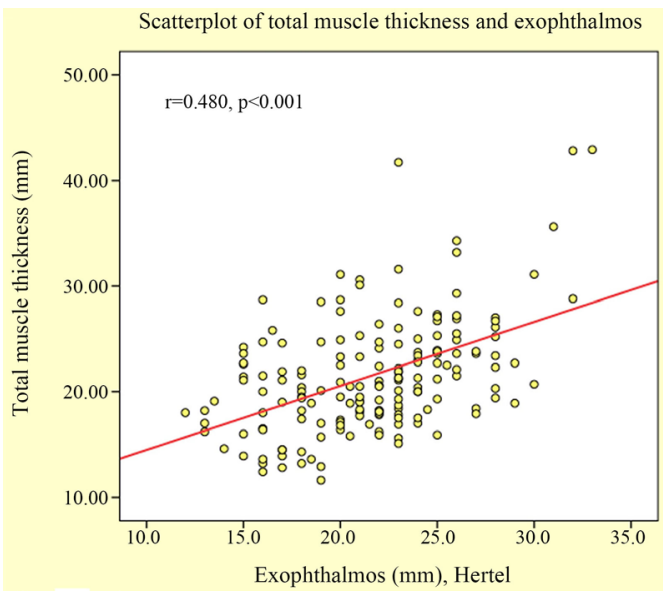

(a)

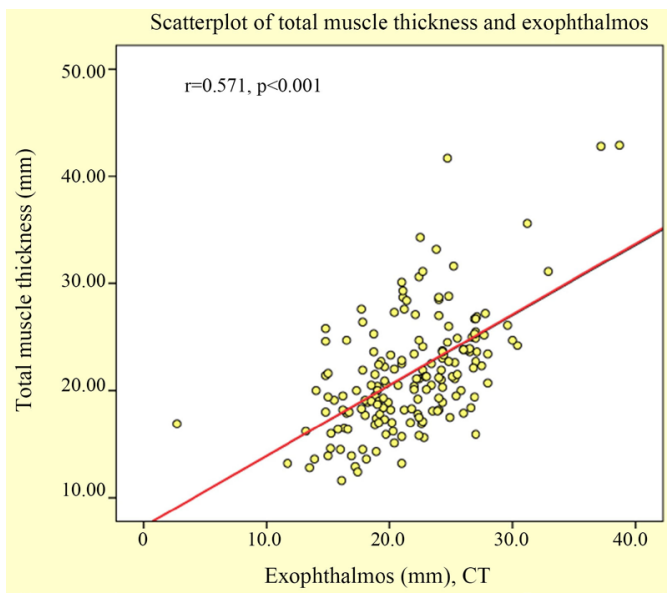

(b)

Figure 2. Significant association between total muscle thickness (muscle thickness sum) and exophthalmos: (a) Hertel exophthalmometry; (b) Computed tomography. 


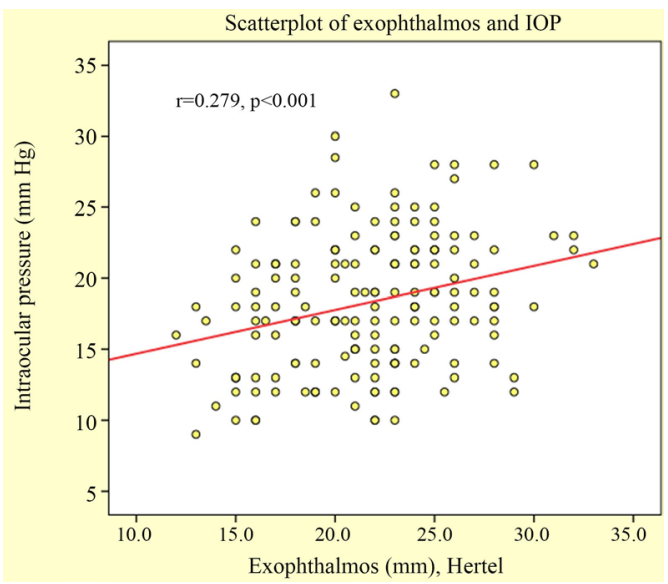

(a)

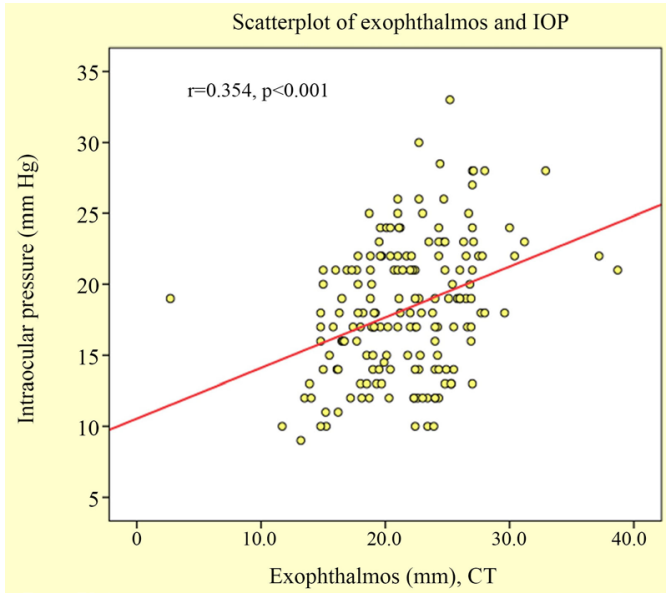

(b)

Figure 3. Significant association between intraocular pressure and exophthalmos: (a) Hertel exophthalmometry; (b) Computed tomography.

Bland-Altman plot

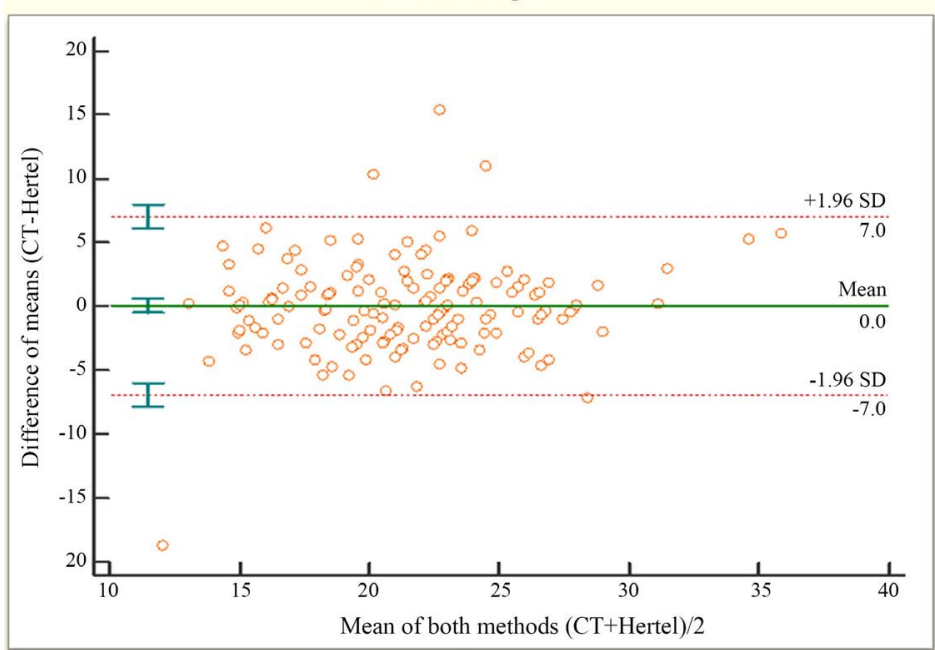

Figure 4. High level of agreement between Computed tomography and Hertel exophthalmometry in exophthalmos measurements illustrated by Bland-Altman plot. CT-Computed tomography; Hertel-Hertel exophthalmometry. 


\section{Discussion}

Exophthalmos is one of the most common objective symptoms in patients with TAO. We established higher values of exophthalmos in men with ophthalmopathy than in women, like in most literature reports. Doric et al. measured mean exophthalmos in males $23.16 \pm 3.61 \mathrm{~mm}$, and in females $-21.42 \pm 3.51 \mathrm{~mm}$, and $\mathrm{Li}$, in a study of Chinese TAO patients, also found higher mean values of proptosis in males (19.94 \pm 3.45$)$ compared to those in women $(18.58 \pm 3.31)$ [4] [5]. In her research Strianese detected a higher number of TAO activity points in exophthalmic eyes ( 3 to 6 , average 4.1 points) than non-exophthalmic eyes ( 2 to 4, 2.8 points average) [6]. In patients with TAO activity, we found higher mean values of exophthalmos compared to patients without disease activity by both methods, which is explained by the swelling and the increase in soft tissue volume of the orbit during an active inflammatory process. The appearance of exophthalmos as an expression of spontaneous decompression in the increased volume of the soft tissues of the orbit is important for both the expansion of the orbital fat and the thickening of the extraocular muscles. Nishida et al. established that increased adipose tissue volume is of greater significance for the development of exophthalmos than enlarged extraocular muscles [7]. Fang discovered that both factors-the increase in orbital fat and the thickening of extraocular muscles play a role in both the protrusion of the eyeball, and the severity and prognosis of the disease [8]. In our study we established a correlation between greater muscle thickness sum and exophthalmos measured by both HE and CT, the patients with disease activity showing greater muscle thickness sum and greater exophthalmos.

A classic method for clinical assessment of the eye protrusion is Hertel exophthalmometry. There are different types of exophthalmometers and there is a certain amount of subjectivity in the reading of the values, which necessitates the correct placement of the device and compliance with all rules when performing the exophthalmometry. Vardizer et al. compared the measurements from 8 different exophthalmometers and found that the design of the devices themselves affects the accuracy of measurement [9]. The authors also considered other factors for correct interpretation of the results, such as a change in the position of the patient's head during the measurement, a change in the direction of sight, incorrect placement of the concave sections of the two frames of the device to the outer rims of the orbits. When monitoring the exophthalmos in a patient with TAO, it is advisable that the measurements are taken by the same physician. Lam et al. studied the differences in the exophthalmometry values of patients with TAO obtained by one and two researchers, and found that there was no significant difference between the results of the same researcher and there is such a difference in the values obtained by different researchers examining the same patient [10]. Another method of estimating proptosis, in which the potential errors in reading values are significantly fewer, is the computed tomography study of the orbits. It determines, on axial scans, the perpendicular distance of the anterior pole of the eye from the interzygomatic line. We established a high 
degree of consistency between Hertel exophthalmometry and multidetector CT when measuring the exophthalmos. Doric et al. also compared the two methods and established a high correlation between the protrusion of the bulb, measured by MDCT and HE, both for the right eye $(r=0.760 ; \mathrm{p}=0.000$; CI 95\%) and the left eye ( $\mathrm{r}=0.799 ; \mathrm{p}=0.000$; CI 95\%) [4]. Ampudia et al. tested 118 orbits of 59 patients with TAO and reported higher levels of exophthalmos measured by Hertel exophthalmometry than by CT, but also found a good correlation between the two methods $(\mathrm{r}=0.72$ for the right eye and $\mathrm{r}=0.65$ for left eye, $\mathrm{p}<$ 0.000 ) [11]. Hauck established slightly lower values of protrusion measured by CT compared to HE and determined an average value of the difference $0.03 \mathrm{~mm}$ [12]. Like Hauck, we measured an absolute value of the difference between the two methods equal to $0.032 \mathrm{~mm}$, but with insignificantly higher CT measurements, and reported a high degree of consistency between the two methods for evaluating exophthalmos. Park et al. compared HE and CT, indicating 3 different methods for CT measuring of the exophthalmos [13] (Figure 5). The first method measures the distance of the cornea to the line joining the two lateral orbital bones on an axial CT slice. The second method measures the exophthalmos of each eye individually along the line that connects the tip of the cornea with the line connecting the lateral and medial orbital rims of each individual orbit on an axial CT slice. The third method uses a sagittal CT slice and takes into account the protrusion along the distance of the cornea from the line that connects the upper and lower orbital rims of each orbit. In all three methods the authors established a high correlation with Hertel exophthalmometry (coefficient of Pearson $r=0.727$ in method $1, r=0.712$ in method 2 , and $r=0.623$ in method 3).

\section{Conclusion}

Exophthalmos is an important clinical feature in patients with TAO, and its measurement and monitoring over time provide an assessment of the clinical course and outcome of the treatment. Hertel exophthalmometry is the most commonly used method of measuring the protrusion of the eyeball, but shows a different reproducibility of results between different researchers. Computed tomography examination of proptosis is easy and should always be used to evaluate orbital CT changes in thyroid-associated ophthalmopathy.
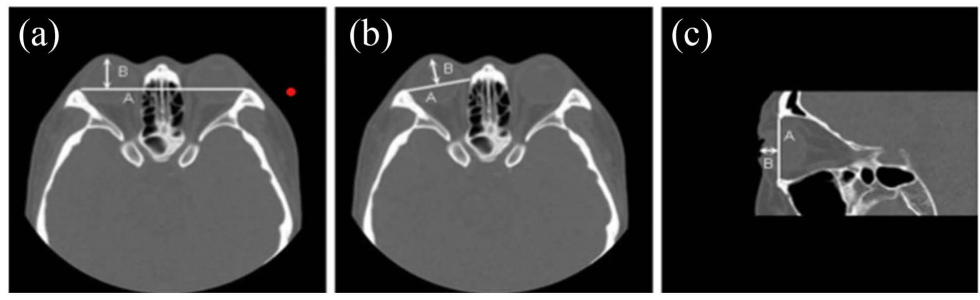

Figure 5. Methods of measuring exophthalmos by CT (Park). (a) By the distance of the cornea B from the interzygomatic line A. (b) By the distance of the cornea B from the line connecting the lateral and medial orbit rims A. (c) By the distance of the cornea $\mathrm{B}$ from the line connecting the upper and lower orbit rims A. 


\section{Conflicts of Interest}

The authors declare no conflicts of interest regarding the publication of this paper.

\section{References}

[1] Petunina, N.A., Trukhina, L.V. and Martirosyan, N.S. (2012) Endocrine Ophthalmopathy-State-of-the-Art Approaches. Problems of Endocrinology, 58, 24-30. https://doi.org/10.14341/probl201258624-32

[2] Mourits, M.P., Prummel, M.F., Wiersinga, W.M. and Koornneef, L. (1989) Clinical Activity Score as a Guide in the Management of Patients with Graves' Ophthalmopathy: A Novel Approach. British Journal of Ophthalmology, 73, 639-644. https://doi.org/10.1136/bjo.73.8.639

[3] Bartalena, L., Baldeschi, L., Dickinson, A., Eckstein, A., Kendall-Taylor, P., Marcocci, C., et al. (2008) Consensus Statement of the European Group on Graves' Orbitopathy (EUGOGO) on Management of GO. European Journal of Endocrinology, 158, 273-285. https://doi.org/10.1530/EJE-07-0666

[4] Đorić, I., Žarković, M., Radojičić, Z., Repac, N., Janićijević, A., Rotim, K., et al. (2017) The Value of Multidetector Computed Tomography of Orbits in Globe Protrusion in Comparison to Hertel Exophthalmometry. Acta Clinica Croatica, 56, 21-27. https://doi.org/10.20471/acc.2017.56.01.04

[5] Li, Q., Ye, H., Ding, Y., Chen, G., Liu, Z., Xu, J., et al. (2017) Clinical Characteristics of Moderate-to-Severe Thyroid Associated Ophthalmopathy in 354 Chinese Cases. PLoS ONE, 12, e0176064. https://doi.org/10.1371/journal.pone.0176064

[6] Strianese, D., Piscopo, R., Elefante, A., Napoli, M., Comune, C., Baronissi, I., et al. (2013) Unilateral Proptosis in Thyroid Eye Disease with Subsequent Contralateral Involvement: Retrospective Follow-Up Study. MBC Ophthalmology, 13, 21. https://doi.org/10.1186/1471-2415-13-21

[7] Nishida, Y., Tian, S., Isberg, B., Hayashi, O., Tallstedt, L. and Lennerstrand, G. (2002) Significance of Orbital Fatty Tissue for Exophthalmos in Thyroid-Associated Ophthalmopathy. Graefe's Archive for Clinical and Experimental Ophthalmology, 240, 515-520. https://doi.org/10.1007/s00417-002-0498-3

[8] Fang, Z.J., Zhang, J.Y. and He, W.M. (2013) CT Features of Exophthalmos in Chinese Subjects with Thyroid-Associated Ophthalmopathy. International Journal of Ophthalmology, 6, 146-149.

[9] Vardizer, Y., Berendschot, T.T. and Mourits, M.P. (2005) Effect of Exophthalmometer Design on Its Accuracy. Ophthalmic Plastic and Reconstructive Surgery, 21, 427-430. https://doi.org/10.1097/01.iop.0000180066.87572.39

[10] Lam, A.K., Lam, C.F., Leung, W.K. and Hung, P.K. (2009) Intra-Observer and Inter-Observer Variation of Hertel Exophthalmometry. Ophthalmic and Physiological Optics, 29, 472-476. https://doi.org/10.1111/j.1475-1313.2008.00617.x

[11] Ampudia, J., Guardia, E., Castrillo, P., Cutillas, M., Soldevila, J. and de Leiva, A. (1993) Thyroid Ophthalmopathy: Clinical and Tomographic Study. Medicina Clínica, 100, 447-449.

[12] Hauck, M.J., Tao, J.P. and Burgett, R.A. (2010) Computed Tomography Exophthalmometry. Ophthalmic Surgery, Lasers \& Imaging, 9, 1-4. https://doi.org/10.3928/15428877-20100210-82

[13] Park, N., Moon, J. and Lee, J. (2019) Hertel Exophthalmometer versus Computed Tomography Scan in Proptosis Estimation in Thyroid-Associated Orbitopathy. Clinical Ophthalmology, 13, 1461-1467. https://doi.org/10.2147/OPTH.S216838 